\title{
Etude analytique de la flore et de la végétation du Parc National de la Ruvubu, Burundi
}

\author{
Tatien MASHARABU ${ }^{1,2}$, Marie José BIGENDAKO ${ }^{2}$, Jean LEJOLY ${ }^{3}$, Jacques \\ NKENGURUTSE $^{2}$, Nausicaa NORET ${ }^{3}$, Elias BIZURU ${ }^{4}$ et Jan BOGAERT ${ }^{1}$ \\ ${ }^{1}$ Université Libre de Bruxelles, Service d'Ecologie du Paysage et Systèmes de Production Végétale, CP 169, 50 \\ Avenue F. Roosevelt, B-1050 Bruxelles, Belgique. \\ ${ }^{2}$ Université du Burundi, Faculté des Sciences, Département de Biologie, B.P. 2700 Bujumbura, Burundi. \\ ${ }^{3}$ Université Libre de Bruxelles, Laboratoire d'Ecologie Végétale et Biogéochimie, CP 244, Boulevard du \\ Triomphe, B-1050 Bruxelles, Belgique. \\ ${ }^{4}$ Université Nationale du Rwanda, Faculté des Sciences, Département de Biologie, B.P.117 Butare, Rwanda. \\ * Auteur correspondant, E-mail: masharabin@yahoo.fr
}

\section{RESUME}

Les études relatives à la flore et à la végétation des aires protégées du Burundi et particulièrement du Parc National de la Ruvubu, la plus grande aire protégée du pays, sont encore fragmentaires. Cette étude se fixe comme objectifs d'individualiser, définir et réaliser une classification syntaxonomique des groupements végétaux de cette aire protégée ainsi que d'évaluer leur structure et diversité. L'étude fournit la liste et la classification des communautés végétales du parc à l'aide de la méthode phytosociologique classique. La richesse spécifique, les indices de diversité de Margalef et de Shannon ainsi que l'équitabilité ont servi à évaluer la diversité et la structure des groupements. Dix groupements variant principalement selon les gradients topographique, pédologique, hydrologique, l'action perturbatrice des feux et le microclimat ont été individualisés. Le statut syntaxonomique des autres unités connues à partir de la littérature a été clarifié. Cinq groupements sont nouveaux: un groupement en savanes (savanes à Hyparrhenia diplandra et Entada abyssinica), deux groupements dans les galeries forestières (galeries forestières à Syzygium cordatum et Aframomum angustifolium, galeries forestières à Syzygium cordatum et Alchornea cordifolia) et deux groupements dans les marais (marais inondés à Cyperus latifolius et Dissotis ruandensis, marais peu inondés à Dissotis brazzae).

(c) 2010 International Formulae Group. All rights reserved.

Mots clés: Analyses multivariées, diversité, groupement végétal, phytosociologie.

\section{INTRODUCTION}

La région de l'Est du Burundi, surtout les savanes et galeries forestières, n'a pas bénéficié d'une attention particulière des chercheurs. La présente étude des phytocénoses du Parc National de la Ruvubu (PNR), la plus grande aire protégée du Burundi, a été réalisée afin de fournir aux différents partenaires de sa gestion un outil de référence sur la variabilité structurale des communautés végétales de cette aire protégée où les travaux relatifs à la flore et à la végétation sont encore fragmentaires (Van Der Ben, 1961 ; Schmitz, 1988 ; Ndabaneze, 1989 ; Vande Weghe et Kabayanda, 1992 ; Masharabu et al., 2008 ; Nzigidahera, 2008 ; Masharabu et al., 2010). En termes de menaces, selon Bigendako et al. (2009), les 
infractions couramment observées dans l'aire protégée sont le braconnage (chasse, piégeage d'animaux sauvages, pêche), la coupe de bois et les feux de brousse. Le PNR constitue néanmoins un sanctuaire et un refuge de biodiversité: mammifères, oiseaux, végétation de savane..... Les savanes d'Afrique sont en effet occupées par la faune de mammifères la plus riche et la plus spectaculaire au niveau mondial. Une bonne proportion est localisée en Afrique de l'Est (Gichohi et al., 1996). Le PNR constitue donc un exemple de cette faune riche d'Afrique orientale unique pour le Burundi. La topographie diversifiée, la géologie, la variabilité spatiale et temporelle du climat en Afrique de l'Est et du Sud ont ainsi créé une mosaïque de types de végétation distincts (Gichohi et al., 1996). Selon Lubini (1982), les inventaires floristiques et l'étude des groupements végétaux constituent l'une des plus importantes sources de données de base notamment pour des recherches portant sur la diversité spécifique, le développement de systèmes de gestion forestière, d'aménagement et de conservation de la nature. On ne peut pas alors prétendre faire de la gestion des réserves naturelles sans connaître la structure, la dynamique et le fonctionnement des phytocénoses. L'approche sociologique basée sur l'affinité des espèces constituantes des phytocénoses est bien indiquée pour la classification des communautés végétales même si, selon Doing (1969), la signification écologique des groupes identifiés paraît difficile à interpréter dans certains cas. Cette étude se fixe comme objectifs d'individualiser, définir et réaliser une classification syntaxonomique des groupements végétaux du PNR ainsi que d'évaluer leurs structures et diversités; l'hypothèse étant que le PNR renfermerait des groupements nouveaux.

\section{MATERIEL ET METHODES} Cette étude s'inspire de la voie déjà
rée dans d'autres travaux phytosociologiques (Lubini, 1980, Habiyaremye, 1997 ; Gillet, 2000, Noumi et Kitio, 2003 ; Bizuru, 2005), qui consiste à utiliser comme unités fonctionnelles les homécies (catégories de synusies de physionomie comparable et occupant la même fonction écologique dans différentes phytocénoses). Les homécies sont ainsi des compartiments homogènes des phytocénoses vis-à-vis des formes biologiques, des types morphologiques végétatifs et des stratégies adaptatives.

Le PNR est en effet situé au Nord-Est du Burundi entre $2^{\circ} 54^{\prime}-3^{\circ} 22^{\prime} \mathrm{S}$ et $30^{\circ} 6^{\prime}-$ $30^{\circ} 33^{\prime}$ E. Il jouit d'un climat tropical de type $\left(\mathrm{AW}_{3}\right)$ s selon la classification de Köppen (1923). L'altitude varie de 1350 à $1830 \mathrm{~m}$. Il s'étend sur une superficie de 50800 ha dont plus de $85 \%$ sont constitués de savanes (Vande Weghe et Kabayanda, 1992 ; Ndabirorere, 1999), le reste étant constitué de galeries forestières et de marais. La Figure 1 montre la visualisation du PNR par rapport au Burundi ainsi que la localisation des axes échantillonnés. Le choix des axes suivis a été guidé par leur représentativité de l'hétérogénéité structurale du PNR. L'échantillonnage a porté sur les bas-fonds, les versants (pentes), les plateaux et les pics des plateaux en tenant compte de l'homogénéité de la station du point de vue floristique et physionomique. Seuls les milieux présentant une apparente uniformité des conditions écologiques ont été retenus. Les données phytosociologiques ont été collectées selon la méthode sigmatiste (Station Internationale de Géobotanique Méditerranéenne et Alpine, SIGMA) classique (BraunBlanquet, 1932) au sein des savanes, galeries forestières et marais. Pour l'inventaire (Tableau 3 or Annexe), la nomenclature des plantes à fleurs a suivi le système de classification de Lebrun et Stork (1991-1997) dont la base des données des plantes à fleurs d'Afrique tropicale est consultable en ligne sur http://www.ville-ge.ch/musinfo/bd/cjb/ 
africa/. La vérification des Pteridophytes s'est référée à Roux (2009).

Les techniques d'analyses multivariées reprises sous le vocable «ordination» facilitent en effet l'analyse d'un tableau de relevés floristiques ou la compréhension des relations entre la végétation et son environnement (Bouxin, 2008); l'ordination pouvant être directe ou indirecte. C'est ainsi que pour individualiser les groupements, le tableau brut des relevés à été soumis au logiciel TWINSPAN (Two-Way Indicator Species Analysis) qui est une méthode de classification divisive, hiérarchique et dichotomique proposée par Hill (1994). Pour apprécier les degrés d'affinité entre les groupements au sein des écosystèmes, un dendrogramme (Cluster analysis) a été par la suite généré à l'aide du logiciel MVSP (MultiVariate Statistical Package) (Kovach, 1997) avec la dissimilitude Bray-Curtis. La dissemblance Bray-Curtis est située entre 0 et 1 , où $0(0 \%)$ signifie que deux sites ont la même composition spécifique et $1(100 \%)$ signifie que deux sites n'ont aucune espèce commune.

Pour donner le statut phytosociologique des différents groupements ou associations identifiés, il a fallu d'abord vérifier s'ils étaient déjà connus dans le système de classification de Schmitz (1988) qui a répertorié toutes les associations décrites valablement pour le Burundi, le Rwanda et la République Démocratique du Congo (R.D. Congo) ou s'ils représentaient de nouveaux syntaxons. Pour ces derniers, il a été décidé de procéder à leur intégration hiérarchique dans les unités syntaxonomiques supérieures (alliance, ordre et classe). L'attribution des noms aux syntaxons nouveaux s'est basée sur les règles détaillées dans le Code International de Nomenclature phytosociologique (Weber et al., 2000). Les groupes écosociologiques utilisés pour caractériser les groupements végétaux de notre zone d'étude sont ceux qui sont repris dans les travaux de Lewalle
(1972), Schmitz (1988) et Bizuru (2005), des auteurs qui ont conduit des études phytosociologiques dans des régions relativement voisines de notre zone d'étude.

Enfin, pour évaluer la diversité des groupements végétaux ainsi que leur structure, la richesse spécifique et les indices de diversité de Margalef $\left(R_{M g}\right)$ (Magurran, 2004), de Shannon $H$ et d'équitabilité de Piélou $E$ (Frontier et al., 2008) ont été utilisés. Ces derniers ont été calculés avec les inventaires floristiques obtenus.

Indice de diversité de Margalef:

$$
R_{M g}=\frac{S-1}{\ln (N)}
$$

avec $S$ nombre d'espèces et $N$ nombre d'individus. La division par $N$ est incluse pour permettre la comparaison entre des communautés présentant un nombre différent d'individus, étant donné que $S \leq N$ d'individus, et constitue une correction de la taille de l'échantillon (Magurran, 2004).

\section{Indice de diversité de Shannon:}

$$
H=-\sum_{i=1}^{s} P_{i} \ln P_{i}
$$

$P_{i}\left(=n_{i} / N\right)$ exprime la probabilité de rencontrer l'espèce $i$ dans le peuplement effectif total $N$. $n_{i}$ correspond au recouvrement moyen de l'espèce $i$ tandis que $N$ équivaut à la somme des recouvrements de l'ensemble des espèces.

\section{Diversité maximale:}

H' $\max =\ln S$. (3)

L'équitabilité $(E)$ correspond à la diversité relative et est définie par la formule suivante:

$E=\frac{H}{H_{\max }^{\prime}}=\frac{H}{\ln S} ;$

avec $S=$ nombre d'espèces.

\section{RESULTATS}

Groupements individualisés et structure

L'analyse $\mathrm{du}$ fichier complet des relevés floristiques montre que les relevés se regroupent avant tout par écosystème. La classification hiérarchique ascendante réalisée 
suivant l'indice de dissimilarité de Bray-Curtis montre une très bonne séparation des groupements par écosystème: quatre groupements et une sous-association en savanes, trois groupements dans les galeries forestières et trois groupements dans les marais (Figure 2). La dissemblance des marais par rapport aux autres écosystèmes est de l'ordre de $96 \%$ tandis qu'elle est de l'ordre de $88 \%$ entre savanes et galeries forestières. Les groupements au sein d'un écosystème ont une dissemblance située entre 60 et $80 \%$.

\section{Groupes écosociologiques et syntaxonomie}

Les groupes écosociologiques utilisés pour caractériser les groupements végétaux de notre zone d'étude sont uniquement limités à trois classes, quatre ordres et sept alliances
(Tableau 1). Les groupes reconnus ici concernent les espèces des savanes non steppiques de la région soudanozambézienne, tant herbeuses qu'arbustives ou boisées (Classe des Hyparrhenietea), les espèces des forêts édaphiques ou espèces des galeries forestières pour le cas du PNR (Classe des Mitragynetea), les végétations herbacées semi-aquatiques des eaux douces et saumâtres des régions chaudes et froides (Classe des Phragmitetea).

\section{Diversité biologique des groupements}

Les valeurs des indices de diversité biologique (richesse spécifique, diversité de Margalef et diversité de Shannon, équitabilité de Piélou) sont fournies dans le tableau 2.

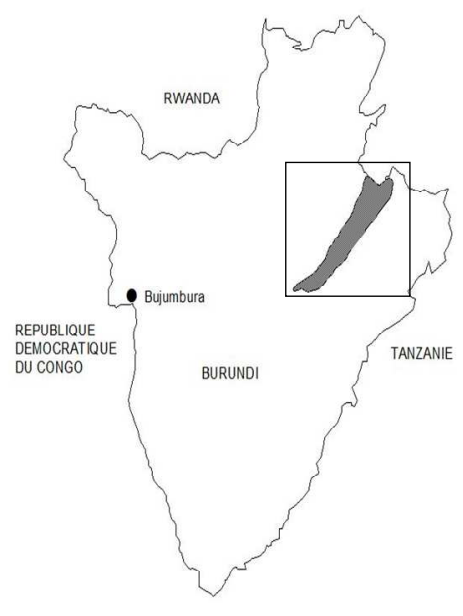

$1 \mathrm{~A}$

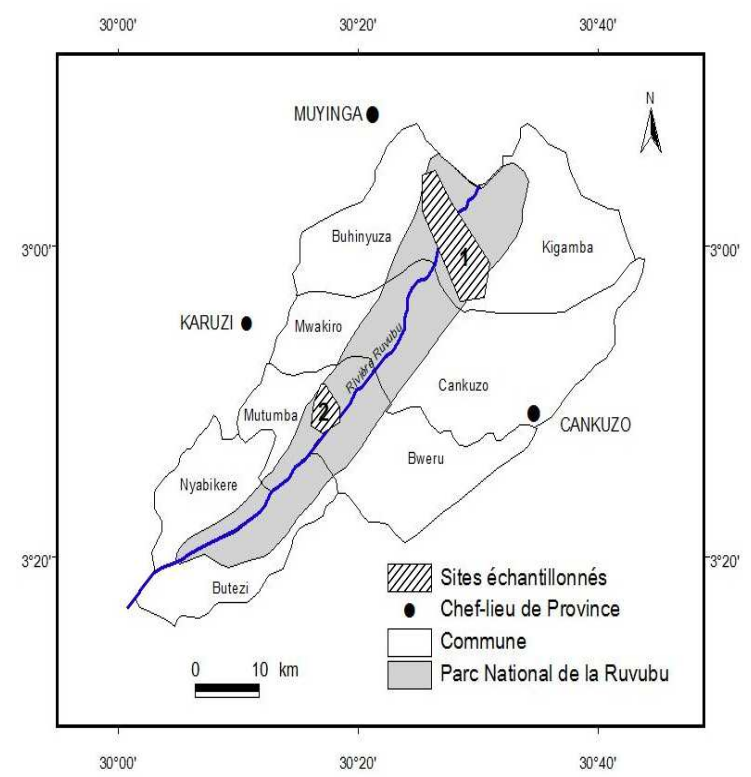

$1 \mathrm{~B}$

Figure 1: 1A. Visualisation du PNR par rapport au Burundi; 1B. Divisions administratives et localisation des axes échantillonnés dans le PNR. Les zones hachurées: (1) représente les sites échantillonnés sur le tronçon Muremera-Gasave. Cette portion se situe entre le bureau du Parc à Muremera, à la rive droite 1 (RD1) (alt. $1671 \mathrm{~m}, 3^{\circ} 3$ ' S, $30^{\circ} 30^{\prime}$ E), commune Kigamba, province Cankuzo et le poste d'entrée de Gasave, à la rive gauche 1 (RG1) (alt. $1507 \mathrm{~m}, 2^{\circ} 56^{\prime} \mathrm{S}, 30^{\circ} 26^{\prime} \mathrm{E}$ ), commune Buhinyuza, province Muyinga; (2) représente les sites échantillonnés à partir du site touristique de Bibara, à la rive gauche 2 (RG2) (alt. $1575 \mathrm{~m}, 3^{\circ} 10^{\prime} \mathrm{S}, 30^{\circ} 17^{\prime} \mathrm{E}$ )., Commune Mutumba, Province Karuzi jusqu'à la rivière Ruvubu (alt. 1380 m, $3^{\circ} 11^{\prime} \mathrm{S}, 30^{\circ} 18^{\prime} \mathrm{E}$ ). 


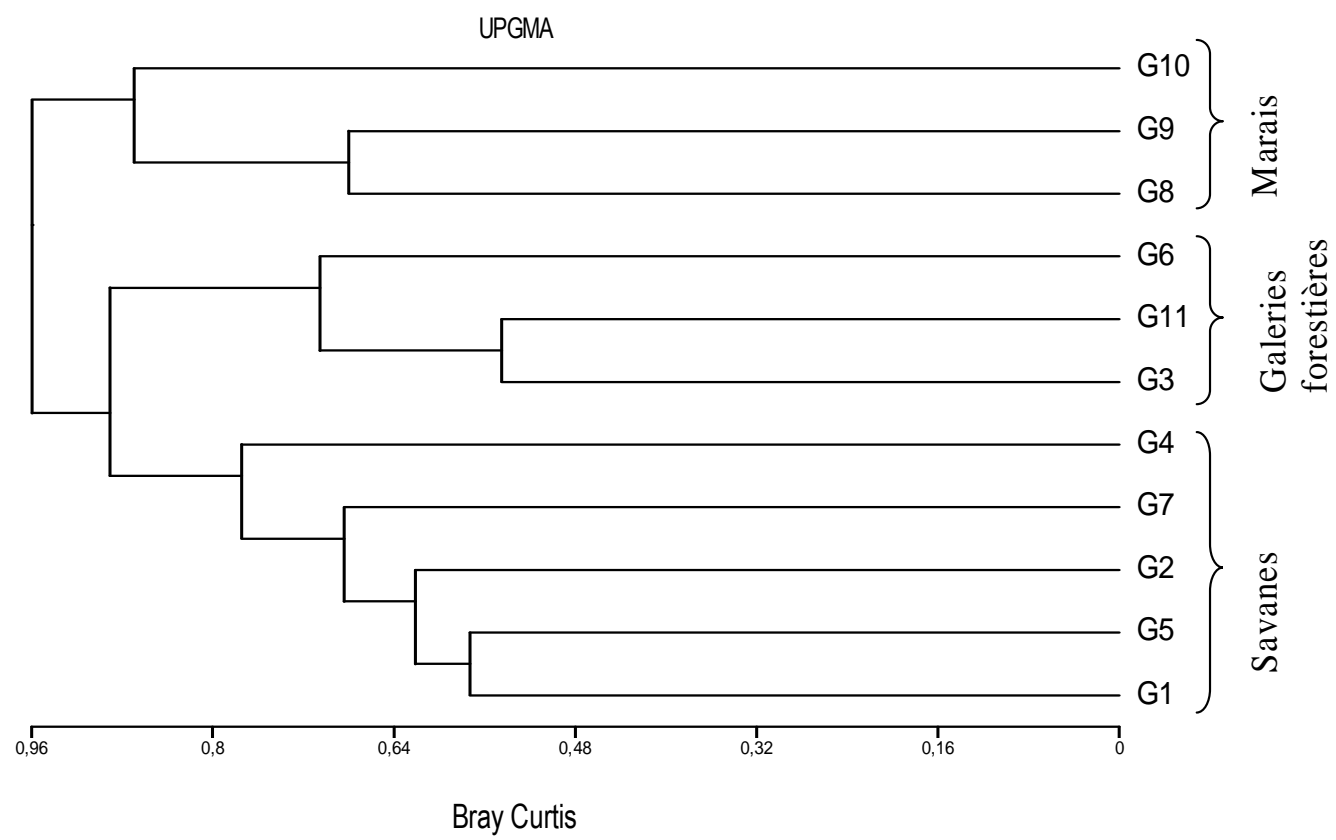

Figure 2: Affinités floristiques entre écosystèmes et groupements individualisés (G1: Groupement à Hyparrhenia diplandra.

G2: Sous-association à Loudetia simplex et Parinari curatellifolia, G3: Groupement à Syzygium cordatum et Alchornea cordifolia, G4: Groupement à Hyparrhenia diplandra et Entada abyssinica, G5: Groupement à Loudetia simplex et Protea madiensis, G6: Groupement à Alchornea cordifolia, G7: Groupement à Loudetia arundinacea et Hymenocardia acida, G8: Groupement à Cyperus latifolius et Dissotis ruandensis, G9: Groupement à Cyperus papyrus et Cyclosorus interruptus, G10: Groupement à Dissotis brazzae, G11: Groupement à Syzygium cordatum et Aframomum angustifolium.

Tableau 1: Groupes écosociologiques et syntaxonomie des communautés végétales individualisées au PNR.

\begin{tabular}{|c|c|c|c|}
\hline Classe & Ordre & Alliance & $\begin{array}{l}\text { Association / Sous- } \\
\text { association }\end{array}$ \\
\hline $\begin{array}{l}\text { Hyparrhenietea } \\
\text { Schmitz } 1963\end{array}$ & $\begin{array}{l}\text { Themedetalia } \\
\text { triandrae Lebrun } \\
1947\end{array}$ & $\begin{array}{l}\text { Hyparrhenion cymbariae } \\
\text { Lebrun } 1947\end{array}$ & $\begin{array}{l}\text { Ass. Loudetia } \\
\text { simplex et Protea } \\
\text { madiensis: Loudetio- } \\
\text { Proteetum madiensis } \\
\text { Lewalle et Schmitz } \\
1988 .\end{array}$ \\
\hline & & & $\begin{array}{l}\text { Sous-ass. Loudetia } \\
\text { simplex et Parinari } \\
\text { curatellifolia: } \\
\text { Loudetio- Proteetum } \\
\text { madiensis } \\
\text { Parinarietosum } \\
\text { Lewalle et Schmitz } \\
1988\end{array}$ \\
\hline
\end{tabular}




\begin{tabular}{|c|c|c|c|}
\hline Classe & Ordre & Alliance & $\begin{array}{l}\text { Association / Sous- } \\
\text { association }\end{array}$ \\
\hline & & $\begin{array}{l}\text { Loudetio- } \\
\text { Euhymenocardion } \\
\text { (Duvigneaud 1949) } \\
\text { (Loudetio- } \\
\text { Euhymenocardienalia } \\
\text { guineensia Duvigneaud } \\
1949 \text { ) }\end{array}$ & $\begin{array}{l}\text { Ass. Hymenocardia } \\
\text { acida et Loudetia } \\
\text { arundinacea: } \\
\text { Hymenocardio- } \\
\text { Loudetietum } \\
\text { arundinaceae } \\
\text { Duvigneaud } 1949\end{array}$ \\
\hline & $\begin{array}{l}\text { Hyparrhenietalia } \\
\text { diplandrae Lebrun } \\
1974\end{array}$ & $\begin{array}{l}\text { Acacio-Hyparrhenion } \\
\text { diplandrae Streel } 1963\end{array}$ & $\begin{array}{l}\text { Ass. Hyparrhenia } \\
\text { diplandra: Loudetio- } \\
\text { Hyparrhenietum } \\
\text { diplandrae A. } \\
\text { Léonard } 1962 \\
\text { Ass. Hyparrhenia } \\
\text { diplandra et Entada } \\
\text { abyssinica: } \\
\text { Hyparrhenio- } \\
\text { Loudetietum } \\
\text { abyssinicae Ass. nov. }\end{array}$ \\
\hline \multirow[t]{2}{*}{$\begin{array}{l}\text { Mitragynetea } \\
\text { Schmitz } 1963\end{array}$} & $\begin{array}{l}\text { Alchornetalia } \\
\text { cordifoliae Lebrun } \\
1947\end{array}$ & $\begin{array}{l}\text { Syzygion cordati Lebrun } \\
1947\end{array}$ & $\begin{array}{l}\text { Ass. Syzygium } \\
\text { cordatum et } \\
\text { Aframomum } \\
\text { angustifolium: } \\
\text { Syzygio } \\
\text { Afromometum } \\
\text { angustifoliae Ass. } \\
\text { nov. } \\
\text { Ass. Syzygium } \\
\text { cordatum et } \\
\text { Alchornea cordifolia: } \\
\text { Syzygio- } \\
\text { Alchorneetum } \\
\text { cordifoliae Ass. nov. }\end{array}$ \\
\hline & & $\begin{array}{l}\text { Alchorneion cordatae } \\
\text { Lebrun } 1947\end{array}$ & $\begin{array}{l}\text { Alchornea cordifolia: } \\
\text { Alchorneetum } \\
\text { cordifoliae Léonard } \\
(1950) 1951\end{array}$ \\
\hline \multirow[t]{2}{*}{$\begin{array}{l}\text { Phragmitetea Tüxen } \\
\text { et Preising } 1942\end{array}$} & $\begin{array}{l}\text { Papyretalia Lebrun } \\
1947\end{array}$ & Papyrion Lebrun 1947 & $\begin{array}{l}\text { Ass. Cyperus papyrus } \\
\text { et Cyclosorus } \\
\text { interruptus: Cypero- } \\
\text { Cyclosoretum } \\
\text { interruptus Comb. } \\
\text { nov. }\end{array}$ \\
\hline & & $\begin{array}{l}\text { Magnocyperion } \\
\text { africanum Lebrun } 1947\end{array}$ & $\begin{array}{l}\text { Ass. Cyperus } \\
\text { latifolius et Dissotis } \\
\text { ruandensis: Cypero- } \\
\text { Dissotietum }\end{array}$ \\
\hline
\end{tabular}




\begin{tabular}{|c|c|c|}
\hline Classe & 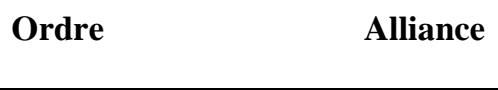 & $\begin{array}{l}\text { Association / Sous- } \\
\text { association }\end{array}$ \\
\hline & & $\begin{array}{l}\text { ruandensis Ass. nov. } \\
\text { Ass. Dissotis } \\
\text { brazzae: Dissotisetum } \\
\text { brazzae Ass. nov. }\end{array}$ \\
\hline
\end{tabular}

Ass.: Association, Sous-ass.: Sous-association, Ass. nov.: Association nouvelle, Comb. nov.: Combinaison nouvelle.

Tableau 2: Diversité biologique des groupements végétaux $\left(R_{M g}\right.$ : Indice de diversité de Margalef, $H$ : Indice de diversité de Shannon, $E$ : Indice d'équitabilité de Piélou, $S$ : Richesse spécifique).

\begin{tabular}{|c|c|c|c|c|c|}
\hline Ecosystèmes & Groupement Sous-/ association & $\boldsymbol{R}_{M g}$ & $\boldsymbol{H}$ & $\boldsymbol{E}$ & $S$ \\
\hline \multirow[t]{6}{*}{ Savanes } & Hyparrhenia diplandra & 23,04 & 2,60 & 0,53 & 133 \\
\hline & $\begin{array}{l}\text { Loudetia simplex et Protea madiensis } \\
\text { Hyparrhenia diplandra et Entada }\end{array}$ & 21,27 & 2,99 & 0,62 & 128 \\
\hline & abyssinica & 20,96 & 2,45 & 0,51 & 124 \\
\hline & $\begin{array}{l}\text { Loudetia arundinacea et Hymenocardia } \\
\text { acida }\end{array}$ & 14,45 & 1.95 & 0,44 & 84 \\
\hline & Loudetia simplex et Parinari & $14,4 \mathrm{~J}$ & 1,93 & 0,44 & 04 \\
\hline & curatellifolia & 14,34 & 1,99 & 0,47 & 71 \\
\hline \multirow[t]{3}{*}{$\begin{array}{l}\text { Galeries } \\
\text { forestières }\end{array}$} & $\begin{array}{l}\text { Syzygium cordatum et Alchornea } \\
\text { cordifolia }\end{array}$ & 18,07 & 2,09 & 0,45 & 103 \\
\hline & Alchornea cordifolia & 11,30 & 2,19 & 0,53 & 65 \\
\hline & $\begin{array}{l}\text { Syzygium cordatum et Aframomum } \\
\text { angustifolium }\end{array}$ & 9,19 & 2,19 & 0,39 & 64 \\
\hline \multirow[t]{4}{*}{ Marais } & Cyperus papyrus et Cyclosorus & & & & \\
\hline & interruptus & 6,75 & 2,19 & 0,61 & 37 \\
\hline & Cyperus latifolius et Dissotis ruandensis & 3,29 & 1,72 & 0,65 & 14 \\
\hline & Dissotis brazzae & 2,15 & 1,99 & 0,91 & 9 \\
\hline
\end{tabular}

Tableau 3: Liste des espèces inventoriées au Parc National de la Ruvubu, Burundi.

\begin{tabular}{ll}
\hline Famille & Espèce \\
\hline DICOTYLEDONES & \\
\hline Acanthaceae & Acanthus pubescens (Thomson ex Oliv.) Engl. \\
& Asystasia gangetica (L.) T.Anderson \\
& Brillantaisia patula T.Anderson \\
& Dicliptera colorata C.B.Clarke \\
& Dyschoriste nobilior C.B.Clarke \\
& Dyschoriste radicans Nees \\
& Dyschoriste trichocalyx (Oliv.) Lindau
\end{tabular}


Hygrophila homblei De Wild.

Hypoestes cancellata Nees

Hypoestes verticillaris (L.f.) Sol. ex Roem. \& Schult.

Isoglossa cyclophylla Mildbr.

Justicia flava (Vahl) Vahl

Justicia matammensis (Schweinf.) Oliv.

Justicia striata (Klotzsch) Bullock

Monechma subsessile (Oliv.) C.B.Clarke

Thunbergia alata Bojer ex Sims

\begin{tabular}{|c|c|}
\hline Amaranthaceae & $\begin{array}{l}\text { Achyranthes aspera } \mathrm{L} . \\
\text { Alternanthera pungens Kunth } \\
\text { Celosia stuhlmanniana Schinz } \\
\text { Cyathula uncinulata (Schrad.) Schinz }\end{array}$ \\
\hline Anacardiaceae & $\begin{array}{l}\text { Lannea edulis (Sond.) Engl. } \\
\text { Lannea schimperi (Hochst. ex A.Rich.) Engl. } \\
\text { Ozoroa reticulata (Baker f.) R.Fern. \& A.Fern. } \\
\text { Pseudospondias microcarpa (A.Rich.) Engl. } \\
\text { Rhus longipes Engl. } \\
\text { Rhus natalensis Bernh. ex C.Krauss } \\
\text { Rhus vulgaris Meikle }\end{array}$ \\
\hline Annonaceae & $\begin{array}{l}\text { Annona senegalensis Pers. } \\
\text { Monanthotaxis orophila (Boutique) Verdc. } \\
\text { Uvaria angolensis Welw. ex Oliv. }\end{array}$ \\
\hline Apiaceae & $\begin{array}{l}\text { Centella asiatica (L.) Urb. } \\
\text { Diplolophium africanum Turcz. } \\
\text { Heteromorpha trifoliata } \text { (H.L.Wendl.) Eckl. \& Zeyh. } \\
\text { Hydrocotyle ranunculoides L.f. } \\
\text { Steganotaenia araliacea } \text { Hochst. }\end{array}$ \\
\hline Apocynaceae & $\begin{array}{l}\text { Adenium obesum (Forssk.) Roem. \& Schult. } \\
\text { Ancylobotrys amoena Hua } \\
\text { Carissa edulis (Forssk.) Vahl } \\
\text { Cryptolepis oblongifolia (Meisn.) Schltr. } \\
\text { Gomphocarpus fruticosus (L.) W.T.Aiton } \\
\text { Landolphia kirkii } \text { R.A.Dyer } \\
\text { Landolphia owariensis } \text { P.Beauv. } \\
\text { Pachycarpus eximius (Schltr.) Bullock } \\
\text { Rhaphionacme decolor Schltr. } \\
\text { Tacazzea apiculata } \text { Oliv. } \\
\text { Voacanga africana } \text { Stapf }\end{array}$ \\
\hline Araliaceae & Cussonia arborea Hochst. ex A.Rich. \\
\hline Asteraceae & $\begin{array}{l}\text { Ageratum conyzoides L. } \\
\text { Anisopappus africanus (Hook.f.) Oliv. \& Hiern } \\
\text { Aspilia ciliata (Schumach.) Wild } \\
\text { Aspilia pluriseta Schweinf. } \\
\text { Athroisma psyllioides (Oliv.) Mattf. } \\
\text { Berkheya spekeana Oliv. } \\
\text { Bidens pilosa } \text { L. } \\
\text { Bidens steppia (Steetz) Sherff } \\
\text { Bothriocline nyungwensis Wech. }\end{array}$ \\
\hline
\end{tabular}


Cirsium buchwaldii O.Hoffm.

Conyza aegyptiaca (L.) Aiton

Conyza subscaposa O.Hoffm.

Conyza sumatrensis (Retz.) E.Walker

Crassocephalum diversifolium Hiern

Crassocephalum multicorymbosum (Klatt) S.Moore

Crassocephalum rubens (Juss. ex Jacq.) S.Moore

Crassocephalum sarcobasis (DC.) S.Moore

Crassocephalum vitellinum (Benth.) S.Moore

Dicoma anomala Sond.

Eclipta prostrata (L.) L.

Elephantopus scaber L.

Emilia caespitosa Oliv.

Emilia humbertii Robyns

Erigeron karvinskianus DC.

Erlangea tomentosa (Oliv. \& Hiern) S.Moore

Erlangea cordifolia (Benth. ex Oliv.) S.Moore

Erlangea longipes (Oliv. \& Hiern) S.Moore

Ethulia conyzoides L.f.

Eupatorium africanum Oliv. \& Hiern

Gerbera ambigua (Cass.) Sch.Bip.

Guizotia scabra (Vis.) Chiov.

Helichrysum keilii Moeser

Helichrysum mechowianum Klatt

Helichrysum nitens Oliv. \& Hiern

Helichrysum nudifolium (L.) Less.

Helichrysum odoratissimum (L.) Sweet

Launaea exauriculata (Oliv. \& Hiern) Amin ex Boulos

Microglossa pyrifolia (Lam.) Kuntze

Mikania capensis DC.

Nidorella spartioides (O.Hoffm.) Cronquist

Piloselloides hirsuta (Forssk.) C.Jeffrey

Pleiotaxis pulcherrima Steetz

Senecio abyssinicus Sch.Bip. ex A.Rich.

Senecio ruwenzoriensis S.Moore

Spilanthes mauritiana (A.Rich. ex Pers.) DC.

Tagetes minuta L.

Taraxacum officinale F.H.Wigg.

Vernonia amygdalina Delile

Vernonia chthonocephala O.Hoffm.

Vernonia fontinalis S.Moore

Vernonia guineensis Benth.

Vernonia lasiopus O.Hoffm.

Vernonia perrottetii Sch.Bip. ex Walp.

Vernonia petersii Oliv. \& Hiern ex Oliv.

Vernonia pogosperma Klatt

Vernonia turbinella S.Moore

Vernonia ugandensis S.Moore 


\begin{tabular}{|c|c|}
\hline Bignoniaceae & $\begin{array}{l}\text { Kigelia africana (Lam.) Benth. } \\
\text { Markhamia obtusifolia (Baker) Sprague } \\
\text { Spathodea campanulata } \text { P.Beauv. }\end{array}$ \\
\hline Bombacaceae & Bombacopsis glabra (Pasq.) A.Robyns \\
\hline Boraginaceae & $\begin{array}{l}\text { Cordia africana Lam. } \\
\text { Trichodesma zeylanicum (Burm.f.) R.Br. }\end{array}$ \\
\hline Caesalpiniaceae & $\begin{array}{l}\text { Caesalpinia decapetala (Roth) Alston } \\
\text { Cassia didymobotrya Fresen. } \\
\text { Cassia kirkii Oliv. } \\
\text { Cassia mimosoides L. } \\
\text { Cassia siamea Lam. } \\
\text { Piliostigma thonningii (Schumach.) Milne-Redh. }\end{array}$ \\
\hline Celastraceae & $\begin{array}{l}\text { Maytenus arbutifolia (Hochst. ex A.Rich.) R.Wilczek } \\
\text { Maytenus arguta (Loes.) N.Robson } \\
\text { Maytenus heterophylla (Eckl. \& Zeyh.) N.Robson } \\
\text { Maytenus senegalensis (Lam.) Exell }\end{array}$ \\
\hline Chrysobalanaceae & $\begin{array}{l}\text { Magnistipula butayei De Wild. } \\
\text { Parinari curatellifolia Planch. ex Benth. }\end{array}$ \\
\hline Clusiaceae & $\begin{array}{l}\text { Garcinia huillensis Welw. ex Oliv. } \\
\text { Harungana madagascariensis Lam. ex Poir. } \\
\text { Psorospermum febrifugum Spach }\end{array}$ \\
\hline Combretaceae & $\begin{array}{l}\text { Combretum binderianum Kotschy } \\
\text { Combretum collinum Fresen. } \\
\text { Combretum molle (Klotzsch) Engl. \& Diels } \\
\text { Terminalia mollis M.A.Lawson }\end{array}$ \\
\hline Convolvulaceae & $\begin{array}{l}\text { Dichondra repens J.R.Forst. \& G.Forst. } \\
\text { Hewittia sublobata } \text { (L.f.) Kuntze } \\
\text { Ipomoea cairica } \text { (L.) Sweet } \\
\text { Ipomoea eriocarpa } \text { R.Br. } \\
\text { Ipomoea involucrata } \text { P.Beauv. } \\
\text { Ipomoea obscura } \text { (L.) Ker Gawl. } \\
\text { Ipomoea rubens } \text { Choisy }\end{array}$ \\
\hline Cucurbitaceae & $\begin{array}{l}\text { Momordica foetida Schumach. } \\
\text { Zehneria scabra (L.f.) Sond. } \\
\text { Zehneria thwaitesii (Schweinf.) C.Jeffrey }\end{array}$ \\
\hline Euphorbiaceae & $\begin{array}{l}\text { Acalypha brachystachya Hornem. } \\
\text { Acalypha hispida } \text { Burm.f. } \\
\text { Acalypha manniana Müll.Arg. } \\
\text { Acalypha ornata Hochst. ex A.Rich. } \\
\text { Acalypha psilostachya Hochst. ex A.Rich. } \\
\text { Acalypha stuhlmannii Pax } \\
\text { Acalypha villicaulis Hochst. ex A.Rich. } \\
\text { Alchornea cordifolia (Schumach. \& Thonn.) Müll.Arg. } \\
\text { Bridelia micrantha (Hochst.) Baill. } \\
\text { Bridelia atroviridis Müll.Arg. } \\
\text { Bridelia brideliifolia (Pax) Fedde } \\
\text { Bridelia scleroneura Müll.Arg. } \\
\text { Clutia abysinica Jaub. \& Spach }\end{array}$ \\
\hline
\end{tabular}


Croton macrostachyus Hochst. ex Delile

Erythrococca bongensis Pax

Euphorbia tirucalli L.

Hymenocardia acida Tul.

Macaranga schweinfurthii Pax

Phyllanthus niruri $\mathrm{L}$.

Phyllanthus ovalifolius Forssk.

Sapium ellipticum (Hochst.) Pax

Synadenium grantii Hook.f.

Tragia brevipes Pax

Uapaca guineensis Müll.Arg.

Uapaca kirkiana Müll.Arg.

Uapaca sansibarica Pax

Fabaceae Aeschynomene elaphroxylon (Guill. \& Perr.) Taub.

Crotalaria aculeata De Wild.

Crotalaria cleomifolia var. seretii De Wild.

Crotalaria glauca var. elliotii Baker f.

Crotalaria lachnophora A.Rich.

Crotalaria lebrunii Baker f.

Crotalaria ononoides Benth.

Crotalaria shirensis (Baker f.) Milne-Redh.

Crotalaria sp Masharabu 594

Crotalaria spartea Baker

Crotalaria spinosa Hochst. ex Benth.

Dalbergia nitidula Baker

Desmodium adscendens (Sw.) DC. var. adscendens

Desmodium barbatum (L.) Benth.

Desmodium ramosissimum G.Don

Desmodium salicifolium (Poir.) DC.

Desmodium setigerum (E.Mey.) Benth. ex Harv.

Desmodium triflorum (L.) DC.

Desmodium velutinum (Willd.) DC.

Dolichos kilimandscharicus Taub.

Dolichos pseudocajanus Baker

Drogmansia pteropus var. pteropus (Baker) De Wild.

Eriosema chrysadenium Taub.

Eriosema lebrunii Staner \& De Craene

Eriosema nutans Schinz

Eriosema psoraleoides (Lam.) G.Don

Eriosema rhodesicum R.E.Fr.

Eriosema stanerianum Hauman

Erythrina abyssinica Lam. ex DC.

Indigofera arrecta Benth. ex Harv.

Indigofera emarginella Steud. ex A.Rich.

Indigofera homblei Baker f. \& Martin

Indigofera paracapitata J.B.Gillett

Indigofera rhynchocarpa Welw. ex Baker

Indigofera spicata Forssk.

Indigofera zenkeri Harms ex Baker f. 
Kotschya aeschynomenoides (Welw. ex Baker) Dewit \& P.A.Duvign.

Kotschya africana var. bequaertii (De Wild.) Verdc.

Kotschya ochreata (Taub.) Dewit \& P.A.Duvign.

Kotschya strigosa (Benth.) Dewit \& P.A.Duvign.

Millettia dura Dunn

Mucuna stans Welw. ex Baker

Neorautanenia mitis (A.Rich.) Verdc.

Pericopsis angolensis (Baker) Meeuwen

Rhynchosia goetzei var. pseudocaribaea Verdc.

Rhynchosia hirta (Andrews) Meikle \& Verdc.

Rhynchosia minima var. macrocalyx (Chiov.) Verdc.

Rhynchosia sublobata (Schumach. \& Thonn.) Meikle Sesbania macrantha Welw. ex E.Phillips \& Hutch.

Sesbania sesban var. nubica Chiov.

Smithia elliotii Baker $\mathrm{f}$.

Sphenostylis marginata E.Mey.

Sphenostylis stenocarpa (Hochst. ex A.Rich.) Harms

Tephrosia linearis (Willd.) Pers.

Tephrosia vogelii Hook.f.

Teramnus labialis (L.f.) Spreng.

Trifolium simense Fresen.

Vigna luteola (Jacq.) Benth.

Vigna parkeri Baker

Flacourtiaceae

Lindackeria bukobensis Gilg

Becium obovatum (E.Mey. ex Benth.) N.E.Br.

Geniosporum rotundifolium Briq.

Haumaniastrum caeruleum (Oliv.) P.A.Duvign. \& Plancke

Hoslundia opposita Vahl

Leonotis nepetifolia (L.) R.Br.

Leucas calostachya Oliv.

Leucas martinicensis (Jacq.) R.Br.

Leucas carsonii Baker

Ocimum basilicum L.

Plectranthus edulis (Vatke) Agnew

Plectranthus defoliatus Hochst. ex Benth.

Plectranthus stachyoides Oliv.

Pycnostachys eminii Gürke

Satureja punctata (Benth.) Briq.

Solenostemon platostomoides (Robyns \& Lebrun) Troupin

Stachys aculeolata Hook.f.

Gentianaceae Anthocleista grandiflora Gilg Anthocleista schweinfurthii Gilg

\begin{tabular}{ll}
\hline Leeaceae & Leea guineensis G.Don \\
\hline Loranthaceae & Tapinanthus constrictiflorus (Engl.) D \\
& Phragmanthera usuiensis (Oliv.) M.G. \\
\hline Malvaceae & Abutilon hirtum (Lam.) Sweet \\
& Hibiscus acetosella Welw. ex Hiern \\
& Hibiscus aponeurus Sprague \& Hutch.
\end{tabular}


Hibiscus diversifolius Jacq.

Hibiscus fuscus Garcke

Pavonia urens Cav.

Sida acuta Burm.f.

Sida alba L.

Sida cordifolia $\mathrm{L}$.

Sida rhombifolia $\mathrm{L}$.

Urena lobata L.

\begin{tabular}{|c|c|}
\hline Melastomataceae & $\begin{array}{l}\text { Antherotoma naudinii Hook.f. } \\
\text { Dissotis brazzae Cogn. } \\
\text { Dissotis ruandensis Engl. } \\
\text { Dissotis senegambiensis (Guill. \& Perr.) Triana } \\
\text { Dissotis trothae Gilg } \\
\text { Tristemma incompletum R.Br. } \\
\text { Tristemma mauritianum J.F.Gmel. }\end{array}$ \\
\hline Meliaceae & $\begin{array}{l}\text { Lepidotrichilia volkensii (Gürke) Leroy } \\
\text { Turraea floribunda Hochst. }\end{array}$ \\
\hline Meliantaceae & Bersama abyssinica Fresen. \\
\hline Menispermaceae & $\begin{array}{l}\text { Cissampelos mисronata A.Rich. } \\
\text { Stephania abyssinica (Quart.-Dill. \& A.Rich.) Walp. } \\
\text { Tinospora caffra (Miers) Troupin }\end{array}$ \\
\hline Mimosaceae & $\begin{array}{l}\text { Acacia hockii De Wild. } \\
\text { Acacia sieberiana DC. } \\
\text { Albizia adianthifolia (Schumach.) W.Wight } \\
\text { Albizia antunesiana Harms } \\
\text { Albizia gummifera (J.F.Gmel.) C.A.Sm. } \\
\text { Entada abyssinica Steud. ex A.Rich. } \\
\text { Mimosa pigra } \text { L. } \\
\text { Newtonia buchananii (Baker f.) G.C.C.Gilbert \& Boutique }\end{array}$ \\
\hline Monimiaceae & Xymalos monospora (Harv.) Baill. \\
\hline Moraceae & $\begin{array}{l}\text { Ficus asperifolia Miq. } \\
\text { Ficus brachypoda Hutch. } \\
\text { Ficus dekdekena (Miq.) A.Rich. } \\
\text { Ficus ingens (Miq.) Miq. } \\
\text { Ficus leprieurii Miq. } \\
\text { Ficus ottoniifolia (Miq.) Miq. } \\
\text { Ficus ovata Vahl } \\
\text { Ficus thonningii Blume } \\
\text { Ficus vallis-choudae Delile } \\
\text { Ficus verruculosa Warb. }\end{array}$ \\
\hline Myristicaceae & Pycnanthus angolensis (Welw.) Warb. \\
\hline Myrsinaceae & $\begin{array}{l}\text { Maesa lanceolata var. golungensis Hiern } \\
\text { Maesa lanceolata var. mildbraedii (Gilg \& G.Schellenb.) } \\
\text { Lebrun } \\
\text { Maesa rufescens A.DC. }\end{array}$ \\
\hline Myrtaceae & $\begin{array}{l}\text { Eugenia malangensis }(\mathrm{O} . \mathrm{Hoffm} .) \text { Nied. } \\
\text { Syzygium cordatum } \text { Hochst. ex C.Krauss } \\
\text { Syzygium guineense (Willd.) DC. subsp. guineense } \\
\text { Syzygium rowlandii Sprague }\end{array}$ \\
\hline
\end{tabular}




\begin{tabular}{|c|c|}
\hline Nymphaeaceae & $\begin{array}{l}\text { Nymphaea lotus L. } \\
\text { Nymphaea maculata Schumach. \& Thonn. }\end{array}$ \\
\hline \multirow[t]{2}{*}{ Ochnaceae } & Ochna holstii Engl. \\
\hline & Ochna schweinfurthiana F.Hoffm. \\
\hline \multirow[t]{2}{*}{ Olacaceae } & Strombosia scheffleri Engl. \\
\hline & Ximenia caffra var. natalensis Sond. \\
\hline \multirow[t]{2}{*}{ Oleaceae } & Jasminum dichotomum Vahl \\
\hline & Schrebera alata (Hochst.) Welw. \\
\hline \multirow[t]{2}{*}{ Onagraceae } & Ludwigia abyssinica A.Rich. \\
\hline & Ludwigia stolonifera (Guill. \& Perr.) P.H.Raven \\
\hline Opiliaceae & Opilia celtidifolia (Guill. \& Perr.) Endl. ex Walp. \\
\hline \multirow[t]{3}{*}{ Oxalidaceae } & Biophytum helenae Buscal. \& Muschl. \\
\hline & Oxalis corniculata $\mathrm{L}$. \\
\hline & Oxalis latifolia Kunth \\
\hline Passifloraceae & Passiflora edulis Sims \\
\hline \multirow[t]{2}{*}{ Pedaliaceae } & Sesamum angolense Welw. \\
\hline & Sesamum angustifolium (Oliv.) Engl. \\
\hline Phytolacaceae & Phytolacca dodecandra L'Hér. \\
\hline \multirow{3}{*}{$\begin{array}{l}\text { Pittosporaceae } \\
\text { Polygalaceae }\end{array}$} & Pittosporum mildbraedii Engl. \\
\hline & Polygala bakeriana Chodat \\
\hline & Securidaca longipedunculata Fresen. \\
\hline \multirow[t]{4}{*}{ Polygonaceae } & Polygonum pulchrum Blume \\
\hline & Polygonum setosulum A.Rich. \\
\hline & Polygonum strigosum R.Br. \\
\hline & Rumex usambarensis (Dammer) Dammer \\
\hline Primulaceae & Lysimachia ruhmeriana Vatke \\
\hline \multirow[t]{4}{*}{ Proteaceae } & Faurea rochetiana (A.Rich.) Chiov. ex Pic. Serm. \\
\hline & Faurea saligna Harv. \\
\hline & Protea bequaertii De Wild. \\
\hline & Protea madiensis Oliv. \\
\hline \multirow[t]{3}{*}{ Ranunculaceae } & Clematis hirsuta Guill. \& Perr. \\
\hline & Clematis simensis Fresen. \\
\hline & Clematopsis scabiosifolia (DC.) Hutch. \\
\hline \multirow[t]{2}{*}{ Rhamnaceae } & Gouania longispicata Engl. \\
\hline & Maesopsis eminii Engl. \\
\hline Rhizophoraceae & Anisophyllea boehmii Engl. \\
\hline Rosaceae & Rubus apetalus Poir. \\
\hline \multirow[t]{11}{*}{ Rubiaceae } & Agathisanthemum globosum (Hochst. ex A.Rich.) Bremek. \\
\hline & Canthium venosum (Oliv.) Hiern \\
\hline & Chassalia subochreata (De Wild.) Robyns \\
\hline & Craterispermum schweinfurthii Hiern \\
\hline & Fadogia ancylantha Schweinf. \\
\hline & Fadogia cienkowskii Schweinf. \\
\hline & Fadogia obovata N.E.Br. \\
\hline & Gardenia ternifolia subsp. jovis-tonantis (Welw.) Verdc. \\
\hline & Hymenodictyon floribundum (Hochst. \& Steud.) Robbr. \\
\hline & Lasianthus kilimandscharicus K.Schum. \\
\hline & Mussaenda arcuata Lam. ex Poir. \\
\hline
\end{tabular}


Oldenlandia herbacea (L.) Roxb.

Oldenlandia lancifolia (Schumach.) DC.

Oldenlandia scopulorum Bullock

Paraknoxia parviflora (Stapf ex Verdc.) Verdc.

Pavetta ternifolia (Oliv.) Hiern

Pavetta virungensis Bremek.

Pentas zanzibarica var. rubra Verdc.

Rubia cordifolia subsp. conotricha (Gand.) Verdc.

Rytigynia kigeziensis Verdc.

Rytigynia castanea Lebrun, Taton \& L.Touss.

Spermacoce dibrachiata Oliv.

Spermacoce natalensis Hochst.

Spermacoce princeae (K.Schum.) Verdc.

Spermacoce senensis (Klotzsch) Hiern

Spermacoce sphaerostigma (A.Rich.) Vatke

Tapiphyllum fadogia Bullock

Virectaria major (K.Schum.) Verdc.

\begin{tabular}{|c|c|}
\hline Rutaceae & Clausena anisata (Willd.) Hook.f. ex Benth. \\
\hline \multirow[t]{4}{*}{ Sapindaceae } & Allophylus macrobotrys Gilg \\
\hline & Blighia unijugata Baker \\
\hline & Dodonaea viscosa Jacq. \\
\hline & Paullinia pinnata $\mathrm{L}$. \\
\hline Sapotaceae & Chrysophyllum gorungosanum Engl. \\
\hline \multirow[t]{2}{*}{ Scrophulariaceae } & Buchnera keilii Mildbr. \\
\hline & Lindernia $s p$ \\
\hline \multirow[t]{6}{*}{ Solanaceae } & Solanum anguivi Lam. \\
\hline & Solanum dasyphyllum Schumach. \& Thonn. \\
\hline & Solanum incanum $\mathrm{L}$. \\
\hline & Solanum nigrum L. \\
\hline & Solanum renschii Vatke \\
\hline & Solanum terminale subsp. sanaganum (Bitter) Heine \\
\hline \multirow[t]{2}{*}{ Sterculiaceae } & Dombeya bagshawei Baker f. \\
\hline & Sterculia tragacantha Lindl. \\
\hline \multirow[t]{4}{*}{ Strychnaceae } & Strychnos cocculoides Baker \\
\hline & Strychnos innocua Delile \\
\hline & Strychnos potatorum L.f. \\
\hline & Strychnos spinosa Lam. \\
\hline \multirow[t]{3}{*}{ Thymeleaceae } & Gnidia ericoides C.H.Wright \\
\hline & Gnidia goetzeana Gilg \\
\hline & Gnidia kraussiana Meisn. \\
\hline \multirow[t]{4}{*}{ Tiliaceae } & Triumfetta cordifolia A.Rich. \\
\hline & Triumfetta flabellato-pilosa R.Wilczek \\
\hline & Triumfetta rhomboidea Jacq. \\
\hline & Triumfetta tomentosa Bojer \\
\hline \multirow[t]{2}{*}{ Ulmaceae } & Celtis africana Burm.f. \\
\hline & Trema orientalis (L.) Blume \\
\hline \multirow[t]{2}{*}{ Urticaceae } & Boehmeria platyphylla Buch.-Ham. ex D.Don \\
\hline & Urtica massaica Mildbr. \\
\hline
\end{tabular}




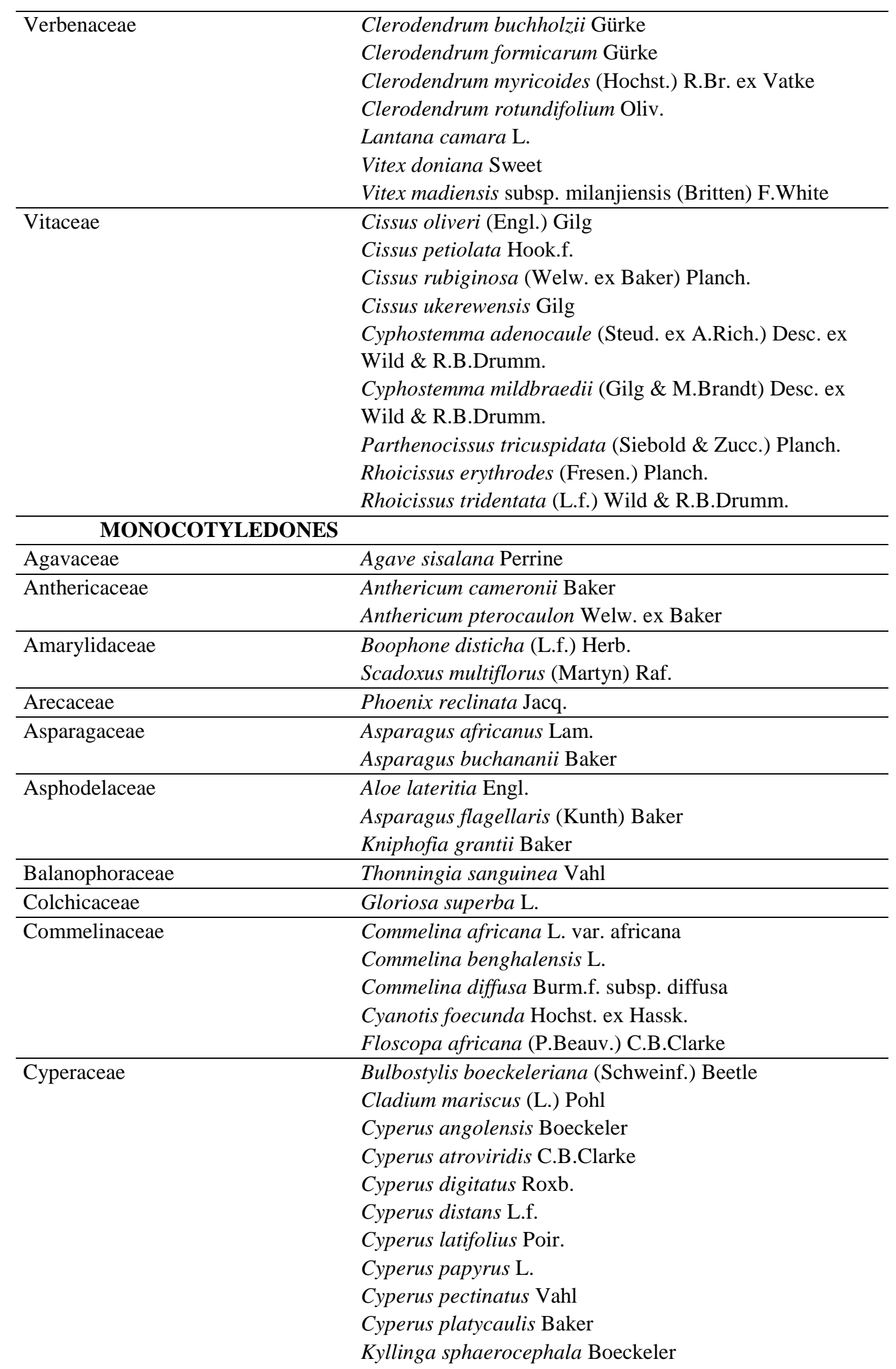


Mariscus macrocarpus Kunth

Mariscus sumatrensis (Retz.) J.Raynal

Scleria nyasensis C.B.Clarke

Scleria racemosa Benth.

\begin{tabular}{|c|c|}
\hline Dioscoreaceae & $\begin{array}{l}\text { Dioscorea alata } \mathrm{L} . \\
\text { Dioscorea bulbifera } \mathrm{L} . \\
\text { Dioscorea dumetorum (Kunth) Pax } \\
\text { Dioscorea odoratissima Pax } \\
\text { Dioscorea quartiniana A.Rich. } \\
\text { Dioscorea schimperiana Hochst. ex Kunth }\end{array}$ \\
\hline Dracaenaceae & Dracaena steudneri Engl. \\
\hline Hyacinthaceae & Albuca abyssinica Jacq. \\
\hline Hypoxidaceae & Curculigo pilosa (Schumach. \& Thonn.) Engl. \\
\hline Iridaceae & $\begin{array}{l}\text { Aristea angolensis } \text { Baker } \\
\text { Gladiolus atropurpureus Baker } \\
\text { Gladiolus dalenii Van Geel }\end{array}$ \\
\hline Orchidaceae & $\begin{array}{l}\text { Brachycorythis pleistophylla } \text { Rchb.f. } \\
\text { Eulophia livingstoniana (Rchb.f.) Summerh. }\end{array}$ \\
\hline Poaceae & $\begin{array}{l}\text { Andropogon schirensis Hochst. ex A.Rich. } \\
\text { Aristida adoensis Hochst. } \\
\text { Arundinella nepalensis Trin. } \\
\text { Brachiaria brizantha (Hochst. ex A.Rich.) Stapf } \\
\text { Ctenium somalense (Chiov.) Chiov. } \\
\text { Cymbopogon caesius (Nees ex Hook. \& Arn.) Stapf } \\
\text { Cymbopogon giganteus Chiov. } \\
\text { Cynodon nlemfuensis } \text { Vanderyst } \\
\text { Digitaria abyssinica } \text { (Hochst. ex A.Rich.) Stapf } \\
\text { Digitaria atrofusca (Hack.) A.Camus } \\
\text { Digitaria longiflora (Retz.) Pers. } \\
\text { Digitaria pearsonii Stapf } \\
\text { Digitaria ternata (A.Rich.) Stapf } \\
\text { Echinochloa pyramidalis (Lam.) Hitchc. \& Chase } \\
\text { Eleusine indica (L.) Gaertn. subsp. indica } \\
\text { Eragrostis capensis (Thunb.) Trin. } \\
\text { Eragrostis exasperata } \text { Peter } \\
\text { Eragrostis olivacea K.Schum. } \\
\text { Eragrostis racemosa (Thunb.) Steud. } \\
\text { Eragrostis tenella (L.) P.Beauv. ex Roem. \& Schult. } \\
\text { Exotheca abyssinica (Hochst. ex A.Rich.) Andersson } \\
\text { Harpachne schimperi Hochst. ex A.Rich. } \\
\text { Hyparrhenia cymbaria (L.) Stapf } \\
\text { Hyparrhenia diplandra (Hack.) Stapf } \\
\text { Hyparrhenia familiaris (Steud.) Stapf } \\
\text { Hyparrhenia filipendula (Hochst.) Stapf } \\
\text { Hyparrhenia newtonii (Hack.) Stapf } \\
\text { Hyparrhenia rufa (Nees) Stapf } \\
\text { Imperata cylindrica (L.) P.Beauv. } \\
\text { Loudetia arundinacea (Hochst. ex A.Rich.) Steud. } \\
\text { Loudetia kagerensis (K.Schum.) C.E.Hubb. ex Hutch. }\end{array}$ \\
\hline
\end{tabular}


Loudetia simplex (Nees) C.E.Hubb.

Melinis minutiflora P.Beauv.

Microchloa kunthii Desv.

Oplismenus compositus (L.) P.Beauv.

Oplismenus hirtellus (L.) P.Beauv.

Panicum adenophorum K.Schum.

Panicum fulgens Stapf

Panicum laxum Sw.

Panicum maximum Jacq.

Panicum phragmitoides Stapf

Paspalum scrobiculatum L.

Pennisetum polystachion (L.) Schult.

Pennisetum purpureum Schumach.

Pennisetum unisetum (Nees) Benth.

Sacciolepis africana C.E.Hubb. \& Snowden

Setaria kagerensis Mez

Setaria megaphylla (Steud.) T.Durand \& Schinz

Setaria pumila (Poir.) Roem. \& Schult.

Setaria sphacelata (Schumach.) Stapf \& C.E.Hubb. ex

M.B.Moss

Sporobolus pyramidalis P.Beauv.

Urelytrum digitatum K.Schum.

\begin{tabular}{ll}
\hline Smilacaceae & Smilax kraussiana Meisn. \\
\hline Xyridaceae & Xyris capensis Thunb. \\
\hline Zingiberaceae & Aframomum angustifolium (Sonn.) K.Schum. \\
& Aframomum zambesiacum (Baker) K.Schum. \\
& Costus spectabilis (Fenzl) K.Schum. \\
\hline \multicolumn{1}{c}{ PTERIDOPHYTES } & \\
\hline Cyatheaceae & Alsophila manniana (Hook.) R.M.Tryon \\
& Alsophila mildbraedii Brause \\
& Alsophila mossambicensis (Baker) R.M.Tryon \\
\hline Dennstaeditiaceae & Histiopteris incisa (Thunb.) J. Sm. \\
& Pteridium aquilinum (L.) Kuhn \\
\hline Dryopteridaceae & Cyclosorus interruptus (Willd.) H.Itô \\
\hline Nephrolepidaceae & Nephrolepis undulata (Afzel. ex Sw.) J.Sm. \\
\hline Osmundaceae & Osmunda regalis L. \\
\hline Pteridaceae & Pteris burtonii Baker \\
\hline
\end{tabular}

Les taxons sont classés par ordre alphabétique des familles, des genres et des espèces au sein des genres en commençant par les classes des Dicotylédones et des Monocotylédones, et enfin les Ptéridophytes. Le système de classification suivi est celui de Lebrun et Stork (1991-1997) pour les Dicotylédones et les Monocotylédones et celui de Roux (2009) pour les Ptéridophytes.

\section{DISCUSSION}

\section{Groupements individualisés et structure}

Après traitement des données, dix groupements végétaux et une sous-association limités à trois classes (Hyparrhenietea, Mitragynetea, Phragmitetea) ont été mis en évidence. Toutefois, il faut signaler la présence des espèces de la classe des
Ruderali-Manihotetea (Taton, 1949) le long des pistes et reposoirs des herbivores (buffles, antilopes, ...): Achyranthes aspera, Ageratum conyzoides, Bidens steppia, Emilia caespitosa, Guizotia scabra, Gutenbergia cordifolia, Leonotis nepetifolia. Il s'agit des espèces caractéristiques des groupements végétaux anthropiques et nitrophiles des zones 
piétinées, des décombres et des bords des routes (Schmitz, 1988).

Parmi les associations végétales, cinq avaient déjà été signalées par Schmitz (1988) au Burundi, Rwanda et R.D. Congo (association à Hyparrhenia diplandra, à Loudetia simplex et Protea madiensis, à Loudetia arundinacea et Hymenocardia acida, à Alchornea cordifolia, à Cyperus papyrus et Cyclosorus interruptus, les cinq autres sont nouvelles.

Bien que discontinus, les groupements identifiés présentent des affinités floristiques selon qu'ils se retrouvent soit dans la même tranche d'altitude ou dans les mêmes conditions hydrologiques ou pédologiques. De cette manière, la distribution des espèces obéit beaucoup plus à la variation du substrat. En général, pour les régions tropicales où la variation altitudinale est faible, c'est la topographie qui est toujours citée comme variable discriminante à laquelle on corrèle le type de sol (Sinsin, 1993) ou l'humidité du sol (Wala, 2004).

Savanes

Les trois groupements et la sousassociation individualisés en savanes sont classés en trois catégories et les gradients topographiques et pédologiques semblent déterminer leur distribution:

Groupements des sommets de plateaux et crêtes latéritiques: ils sont situés entre 1525 et $1830 \mathrm{~m}$ d'altitude:

- Groupement à Loudetia simplex et Protea madiensis: Loudetio-Proteetum madiensis (Lewalle et Schmitz, 1988): les principales espèces caractéristiques sont: L. simplex, P.madiensis, Anisophyllea boehmii, Tapinanthus constrictiflorus, Gnidia kraussiana et Sphenostylis marginata. Ce groupement se rencontre sur les plateaux latéritiques dans notre dition. Les relevés de ce groupement ont été effectués à des altitudes variant entre 1560 et $1600 \mathrm{~m}$. Les arbustes $P$. madiensis, Strychnos spinosa et Vitex madiensis sont éparpillés dans le tapis graminéen des savanes herbeuses à L. simplex. Schmitz (1988) signale que L. simplex, Rhynchelytrum repens, Cymbopogon afronardus, Hyparrhenia filipendula, $P$. madiensis, Indigofera asparagoides, Helichrysum mechowianum comptent parmi les espèces habituelles. Selon le type de sol, la pente, l'altitude et la végétation forestière ambiante, cet auteur propose une série de sous-associations et groupements divers dont la sous-association à Parinari curatellifolia subsp. mobolla (Loudetio-Proteetum madiensis Parinarietosum (Lewalle et Schmitz, 1988) avec également Anisophyllea boehmii, Strychnos spinosa, Indigofera podocarpa... Cette dénomination est aussi valable ici dans la mesure où on observe parfois au sein du groupement à Loudetia simplex et Protea madiensis des îlots de Parinari curatellifolia. Cette sous-association à Loudetia simplex et Parinari curatellifolia identifiée au PNR est proche de celle proposée par Schmitz (1988). Elle se rencontre sur des sols latéritiques dans la même gamme d'altitude. Elle semble avoir une prédilection sur les sols squelettiques des sommets de plateaux et sur les crêtes latéritiques. Ses principales espèces indicatrices sont: $L$. simplex, P. curatellifolia, Ozoroa reticulata, Pleiotaxis pulcherrima, Synadenium grantii et Ficus ovata.

Groupements des versants (pentes de plateaux ou piémonts): ils sont situés entre 1475 et $1525 \mathrm{~m}$ d'altitude:

- Groupement à Loudetia arundinacea et Hymenocardia acida: HymenocardioLoudetietum arundinacea (Duvigneaud, 1949): il s'établit sur les pentes de plateaux et sur des sols relativement peu profonds. Cette association est une savane arbustive dont la dense strate herbacée est quasiment dominée par L. arundinacea. La forme de cette végétation est due à la nature du sol et aux feux. Les troncs d'arbres sont carbonisés mais leur taille est relativement plus grande que ceux de l'association à $L$. simplex et P.madiensis suite à la clémence du sol. Les espèces caractéristiques sont $L$. arundinacea, H. acida, Justicia flava, Crotalaria spinosa, Cassia mimosoides et Vernonia turbinella. Dans certaines stations, on trouve des faciès à Panicum maximum avec une couverture clairsemée d'H. acida, sur des sols relativement plus fertiles.

Groupements des plateaux et bas-fonds: ils sont situés entre 1400 et 1475 m d'altitude: - Groupement à Hyparrhenia diplandra: Loudetio-Hyparrhenietum diplandrae (Léonard, 1962): il se retrouve sur des sols fertiles qu'occupaient jadis les exploitations agricoles et les habitations avant la création du parc en 1980. Le constat de Schmitz (1988) 
est aussi valable dans notre cas: «Il s'agit de petites stations étrangères à la savane environnante ». Il constitue une zone de transition entre la savane et les galeries forestières. Par endroits, elle est en continuité avec les galeries forestières. Cette végétation constitue un lieu privilégié de pâturage des herbivores essentiellement les buffles qui freinent l'expansion des galeries forestières et influencent sa physionomie et sa composition floristique typique de savane. Cela explique d'ailleurs la présence de beaucoup d'espèces nitrophiles, rudérales et postculturales notamment Achyranthes aspera, Ageratum conyzoides, Asystasia gangetica, Cyathula uncinulata, Guizotia scabra. Les principales espèces caractéristiques sont H.diplandra, Acanthus pubescens, Annona senegalensis, Berkheya spekeana, Senecio ruwenzoriensis, Panicum phragmitoides, Erythrina abyssinica. Notons également la présence des espèces qui révèlent la présence ancienne de l'homme comme Panicum phragmitoides, Ficus ingens, F. ovata, Erythrina abyssinica, Dracaena steudneri, des essences à signification socioculturelle (Masharabu et al., 2008).

- Groupement à Hyparrhenia diplandra et Entada abyssinica: ce groupement a été observé sur un substrat non latéritique, sur des sols meubles et parfois caillouteux à des altitudes situées autour de $1600 \mathrm{~m}$. Les troncs d'E. abyssinica sont carbonisés, ce qui révèle que ce groupement est annuellement parcouru par les feux. Les espèces indicatrices sont $H$. diplandra, E. abyssinica et $H$. cymbaria.

\section{Galeries forestières}

Le gradient topographique semble influencer la distribution des trois groupements individualisés dans les galeries forestières:

- Groupement à Syzygium cordatum et Aframomum angustifolium: ce groupement s'observe à des altitudes situées entre 1500 et $1600 \mathrm{~m}$. Les principales espèces indicatrices du groupement sont $S$. cordatum, A. angustifolium, Thunbergia alata, Phyllanthus niruri, Dodonaea viscosa et Triumfetta flabellato-pilosa. La strate arborée est dominée par $S$. cordatum tandis que la strate herbacée est largement dominée par $A$. angustifolium. Cette dernière espèce se rencontre souvent dans les endroits humides des galeries forestières ou bien dans les zones de contact entre savanes sur sols humides et galeries forestières.

- Groupement à Syzygium cordatum et Alchornea cordifolia: ce groupement se rencontre à des altitudes situées autour de $1500 \mathrm{~m}$ dans des stations longeant les cours d'eau. La strate arborée est quasiment dominée par $S$. cordatum. Les espèces indicatrices sont: S. cordatum, A. cordifolia, Rhus vulgaris et Cassia kirkii.

- Groupement à Alchornea cordifolia:
Alchorneetum cordifoliae (Léonard, 1950,1951): cordons et franges arbustifs sur substrat toujours très humide et périodiquement inondé, des diverses régions à climat guinéen et voisines (Schimtz, 1988). Ce groupement se rencontre aux environs de $1350 \mathrm{~m}$ d'altitude le long des cours d'eau. Quelques pieds de Phoenix reclinata sont remarquables au niveau de la strate arborée. Les principales espèces indicatrices de ce groupement sont A. cordifolia, Heteromorpha trifoliata, Steganotaenia araliacea et Vernonia amygdalina.

Marais

Les facteurs hydrologiques (profondeur de l'eau) et pédologiques (apport en matières organiques charriées par les eaux de ruissellement) expliqueraient la distance entre les trois communautés végétales des marais:

- Groupement à Cyperus latifolius et Dissotis ruandensis: ce groupement a été observé dans les stations situées à la périphérie des marais à Cyperus papyrus, au contact des collines avoisinantes. Il se développe dans des milieux vaseux et inondés, bénéficiant des apports de matériaux organiques charriés par les eaux de ruissellement des piémonts. Les principales espèces caractéristiques sont C. latifolius, Dissotis ruandensis, C. digitatus, C. distans, Mariscus sumatrensis, Nymphaea maculata et Virectaria major.

- Groupement à Cyperus papyrus et Cyclosorus interruptus: il s'agit du CyperoDryopteridetum gongylodis (Germain, 1952). Cyclosorus interruptus et Dryopteris gongylodes sont des synonymes; $C$. interruptus étant le nom valide (Roux, 2009). Il forme d'immenses prairies flottantes. Cette association est, comme dans les autres régions où elle a été signalée, dominée par Cyperus papyrus et Cyclosorus interruptus. Les principales espèces indicatrices sont: Cyperus papyrus, Cyclosorus interruptus, Dissotis 
trothae et Echinochloa pyramidalis. C'est une association qui a été bien documentée dans différentes régions d'Afrique centrale et orientale (Germain, 1952 ; Mullenders, 1954 ; Van Der Ben, 1961; Gaudet, 1975 ; Thompson et al., 1979 ; Jones et Muthuri, 1985 ; Jolly et Bonnefille, 1992; Bizuru, 2005 ; Dushimirimana et al., 2010). Il est donc possible d'analyser les variations géographiques de cette association. Elle a été décrite pour la première fois dans la partie congolaise de la plaine de la Rusizi par Germain (1952), et puis par Van Der Ben (1961) dans le bassin de Karuzi au Burundi, et récemment par Bizuru (2005) et Dushimirimana et al. (2010) en moyenne altitude dans le Nord du Burundi. Mullenders (1954) fait observer que les papyraies des actuels centres d'endémisme guinéo-congolais et zambéziens sont floristiquement pauvres par rapport aux peuplements de la mosaïque régionale du Lac Victoria. De façon générale, les marais de moyenne altitude sont dominés par C. papyrus, espèce caractéristique du bassin du Nil depuis sa source jusqu'en Egypte; la rivière Ruvubu étant sa source la plus méridionale.

- Groupement à Dissotis brazzae: ce groupement a été observé aux abords du pont reliant les provinces Muyinga et Cankuzo sur la Route Nationale 19 (RN19). Il présente des faciès à Nymphaea maculata par endroits et particulièrement dans les espaces enregistrant une grande profondeur d'eau. Les principales espèces indicatrices sont: Dissotis brazzae, Mimosa pigra, Ludwigia abyssinica, $P$. setosulum et $N$. maculata.

\section{Diversité biologique des groupements}

Selon certains auteurs (Mason, 1977), l'usage des indices de diversité de communautés végétales rencontrées dans des sites différents ou d'une même communauté rencontrée sur des sites variés ou étudiée à des périodes différentes permet de tirer le maximum d'informations écologiques. La richesse spécifique des savanes, des galeries forestières et des marais du PNR est respectivement évaluée à 270 espèces, 146 espèces et 43 espèces (Masharabu et al., sous presse). Dans les marais de Nyamuswaga au Nord du Burundi, Dushimirimana et al. (2010) ont inventorié 63 espèces; un cortège floristique qui ne s'écarte pas beaucoup de celui des marais du PNR.

$\mathrm{Au}$ sein des savanes, l'association à Hyparrhenia diplandra est la plus diversifiée suite aux conditions topographiques et pédologiques favorables, aux déjections des animaux et à l'action perturbatrice de ceux-ci. La pauvreté relative des groupements à Loudetia arundinacea et Hymenocardia acida d'une part et de la sous-association à Loudetia simplex et Parinari curatellifolia d'autre part serait essentiellement due à la nature du sol. Ces deux derniers groupements s'établissent sur des sols écologiquement rudes.

$\mathrm{Au}$ sein des galeries forestières, le groupement à Syzygium cordatum et Alchornea cordifolia est le plus diversifié par rapport aux autres groupements de la même catégorie. Les facteurs topographiques, pédologiques et hydrologiques constitueraient un groupe de facteurs discriminants au sein de ce type de végétation.

$\mathrm{Au}$ sein des marais, les principaux facteurs abiotiques influant sur la diversité des divers groupements seraient la profondeur de l'eau et la nature du sol. Le groupement à Cyperus papyrus et Cyclosorus interruptus est le plus diversifié tandis que le groupement à Dissotis brazzae est le plus pauvre, ce dernier étant submergé. Dans les marais de moyenne altitude au Burundi, Bizuru (2005) a montré que l'association à Cyperus papyrus et Cyclosorus interruptus (=Dryopteris gongylodes) a une richesse spécifique de 58 espèces avec un indice de diversité de Shannon d'une valeur de 2,4 et un indice de régularité de Piélou s'élèvant à 0,40. Dans les marais de Nyamuswaga (Burundi), le facteur discriminant pour ce groupement est la profondeur de l'eau (Dushimirimana et al., 2010).

De façon générale, les groupements végétaux du PNR présentent une diversité taxonomique relativement faible étant donné que l'indice de diversité maximale pour 133 espèces (cas de l'association à Hyparrhenia diplandra) et neuf espèces (cas du groupement à Dissotis brazzae) est évalué respectivement à 4,89 et 2,2. D'autre part, les tendances de l'indice d'équitabilité de Piélou laissent penser à des communautés végétales perturbées. En effet, l'équitabilité (régularité ou encore d'équirépartition chez certains écologues) varie de 0 à 1 . Elle tend vers zéro 
lorsqu'une espèce domine largement le peuplement et elle est égale à un lorsque toutes les espèces ont la même abondance. Un indice d'équitabilité inférieur à $60 \%(<0,6)$ caractérise un environnement perturbé (Diop, 1999). En définitive, le PNR est sujet à des perturbations diverses nécessitant des mesures de mitigation.

\section{REMERCIEMENTS}

Les auteurs adressent leurs remerciements au Gouvernement du Burundi et au Fonds Alice \& David Van Buuren, respectivement pour la bourse d'étude et les subsides Van Buuren octroyés à Masharabu T., à l'Institut National (Burundi) pour l'Environnement et la Conservation de la Nature (INECN) pour la collaboration de son personnel affecté sur le terrain au Parc National de la Ruvubu ainsi qu'aux examinateurs $\mathrm{du}$ manuscrit pour leur contribution dans son amélioration.

\section{BIBLIOGRAPHIE}

Bigendako M J, Gapusi J R, Masharabu T. 2009. Connaissances actuelles, expériences et potentialités des espèces ligneuses autochtones du Burundi. ACVE et EGP-IUCN Netherlands. Bujumbura.

Bizuru E. 2005. Etude de la flore et de la végétation des marais du Burundi. Thèse de doctorat, Université Libre de Bruxelles, p. 311.

Bouxin G. 2008. Analyse statistique des données de végétation. Les techniques d'analyse factorielle et d'ordination. [en ligne]: http://users.skynet.be/Bouxin.Guy/ASDV /ASDV.htm.

Braun-Blanquet J. 1932. Plant sociology. The Study of Plant Communities. Mac Gray Hill (Ed): New York, London.

Diop M S. 1999. Diversité aviaire et comportement reproducteur des Petits Calaos à bec rouge (Tockus erythrorhynchus) et à bec noir (Tockus nasutus) dans les aires protégées de la Petite-Côte du Sénégal.Thèse de doctorat, Université Cheikh Anta Diop de Dakar, p. 187.

Doing H. 1969. Sociological species groups. Acta botanica Neerlandica, 18: 398-400.

Dushimirimana S, Masharabu T, Bizuru E, Bigendako M J. 2010. Flore et végétation naturelle des marais de Nyamuswaga, Burundi. Bulletin Scientifique de l'INECN, 8: 10-15.

Frontier S, Pichod-Viale D, Leprêtre A, Davoult D, Luczak C. 2008. Ecosystèmes: Structure, Fonctionnement, Evolution (4 ${ }^{\grave{e}}$ édition). Dunod : Paris.

Gaudet JJ. 1975. Mineral Concentrations in Papyrus in various African Swamps. Journal of Ecology, 63(2): 483-491.

Germain R. 1952. Les associations végétales de la plaine de la Rusizi (Congo belge) en relation avec le milieu. Publication de l'INEAC, Série Scientifique, 52: 321.

Gichohi H, Gakahu C, Mwangi E. 1996. Savanna ecosystems. In East African Ecosystems and their Conservation, McClanahan TR, Young TP (eds). Oxford University Press: New York; 273298.

Gillet F. 2000. La Phytosociologie synusiale intégrée - Guide méthodologique $\left(4^{\text {ème }}\right.$ édition revue et corrigée). Documents du Laboratoire d'Ecologie Végétale, 1: 68 p. Université de Neuchâtel-Institut de Botanique.

Habiyaremye MKFX. 1997. Etude phytocoenologique de la dorsale orientale du Lac Kivu (Rwanda). Musée Royal de l'Afrique Centrale, Tervuren (Belgique). Annales Sciences Economiques, 24: 276.

Hill MO. 1994. DECORANA and TWINSPAN, for ordination and classification of multivariate species data: a new edition, together with supporting programs, In FORTRAN 77, TABLEFIT Programs (ed.). Huntington, England.

Jolly D, Bonnefille R. 1992. Histoire et dynamique du marécage tropical de Ndurumu (Burundi), données polliniques. Review of Palaeobotany and Palynology, 75: 133-151.

Jones MB, Muthuri FM. 1985. The canopy structure and microclimate of Papyrus (Cyperus papyrus) swamps. Journal of Ecology, 73(2): 481-491.

Köppen W. 1923. Die Klimate der Erde Grundriss der Klimakunde. Walter de Gruyter \& Co.: Berlin, Leipzig.

Kovach WL. 1997. MVSP: MultiVariate Statistical Package Data Analysis. Kovach Computing Services; 85 Nant-yFelin, Pentraeth, Anglesey. 
Lebrun JP, Stork AL. 1991-1997. Enumération des plantes à fleurs d'Afrique tropicale. Conservatoire et Jardin Botanique de la ville de Genève, 4 volumes.

Lewalle J. 1972. Les étages de végétation du Burundi occidental. Bulletin du Jardin Botanique National de Belgique, 42(1/2): 1-247.

Lubini A. 1982. Végétation messicole et postculturale des sous-régions de Kisangani et de la Tshopo (Haut-Zaïre). Thèse de doctorat, Université de Kisangani, p. 489.

Lubini A. 1980. Etude analytique du groupement messicole à Spermacoce latifolia dans la région de Kisangani (Zaïre). Bulletin du Jardin Botanique National de Belgique, 50: 123-133.

Magurran AE. 2004. Measuring Biological Diversity. Blackwell Publishing: Oxford.

Masharabu T, Noret N, Lejoly J, Bigendako MJ, Bogaert J. (Sous presse). Etude comparative des paramètres floristiques du Parc National de la Ruvubu, Burundi. Geo-Eco-Trop.

Masharabu T, Noret N, Lejoly J, Bigendako M J, Bogaert J. 2010. Distribution et fréquence d'occurrence des plantes vasculaires au Parc National de la Ruvubu, Burundi. European Journal of Scientific Research, 43(4): 466-479.

Masharabu T, Lejoly J, Bigendako MJ, Bogaert J, Godart M-F. 2008. Diversité floristique du Parc National de la Ruvubu (Burundi). Bulletin Scientifique de l' INECN, 6: 2-7.

Mason CF. 1977. The performance of a diversity index in describing the zoobenthos of two lakes. Journal of Applied Ecology, 14: 363-367.

Mullenders W. 1954. La végétation de Kaniama (entre Lubishi-Lubilash, Congo belge). Publications de l'INEAC, série scientifique, 61: 499 p. +27 photos.

Ndabaneze P. 1989. Catalogue des Graminées $d u$ Burundi. Les Editions de Lejeunia, Nouvelle série, 132: 127 p.+Annexes.

Ndabirorere S. 1999. Données statistiques des produits forestiers non-ligneux du Burundi. Programme de partenariat CEFAO (1998-2001). Ligne budgétaire forêt tropicale B7-6201/97-15/VIII/FOR PROJET GCP/INT/679/EC.

Noumi E, Kitio E. 2003. Association semiaquatique à Cyclosorus striatus (Schum.) Ching (Thelipteridaceae) dans la région de Yaoundé (Cameroun). African Journal of Science and Technology, 4(2): 29-37.

Nzigidahera B. 2008. S'alimenter en savanes de l'Est du Burundi: Plantes comestibles du Parc National de la Ruvubu. Bulletin Scientifique de l' INECN, 5: 18-23.

Roux JP. 2009. Synopsis of the Lycopodiophyta and Pteridophyta of Africa, Madagascar and neighbouring islands. South African National Biodiversity Institute. Strelitzia, 23: 296 p.

Schmitz A. 1988. Révision des groupements végétaux décrits du Zaïre, du Rwanda et du Burundi. Musée royal de l'Afrique Centrale, Tervuren (Belgique). Annales Sciences Economiques, 17: 315 p.

Sinsin B. 1993. Phytosociolgie, écologie, valeur pastorale, production et capacité de charge des pâturages du périmètre NikkiKalalé au Nord-Bénin. Thèse de doctorat, Université Libre de Bruxelles, p. 350.

Thompson K, Shewry PR, Woolhouse HW. 1979. Papyrus swamp development in the Upemba basin, Zaïre: studies of population structure in Cyperus papyrus stands. Botanical Journal of the Linnean Society, 78: 299-316.

Van Der Ben D. 1961. Phytosociologie. Archives de la mission de la Karuzi (Urundi). Service des Publ. Ass. Tech. Min. Aff. Etrangères et Com. Ext., Bruxelles, 1: 109-133.

Vande Weghe J.P., Kabayanda A. 1992. Le Parc National de la Ruvubu et sa région limitrophe:étude d'identification de la Ruvubu: Etude $N^{\circ}$ ET/44/2/92.

MINATE-CEE Wala K. 2004. La végétation de la chaîne de l'Atakora au Bénin: diversité floristique, phytosociologie et impact humain. Thèse de doctorat, Université de Lomé, p.139.

Weber HE, Moravec J, Theurillat J-P. 2000. International code of phytosociological nomenclature (3rd edn). Journal of Vegetation Science, 11: 739-768. 